\title{
Object recognition memory and the rodent hippocampus
}

\author{
Nicola J. Broadbent, ${ }^{1}$ Stephane Gaskin, ${ }^{2}$ Larry R. Squire, ${ }^{1,3,4,5}$ and Robert E. Clark ${ }^{1,3,6}$ \\ ${ }^{1}$ Department of Psychiatry, University of California, La Jolla, California 92093, USA; ${ }^{2}$ Department of Psychology, Concordia \\ University, Montreal, Quebec H4B 1R6, Canada; ${ }^{3}$ Veterans Affairs Medical Center, San Diego, California 92161, USA; \\ ${ }^{4}$ Department of Neurosciences, University of California, La Jolla, California 92093, USA; ${ }^{5}$ Department of Psychology, University of \\ California, La Jolla, California 92093, USA
}

\begin{abstract}
In rodents, the novel object recognition task (NOR) has become a benchmark task for assessing recognition memory. Yet, despite its widespread use, a consensus has not developed about which brain structures are important for task performance. We assessed both the anterograde and retrograde effects of hippocampal lesions on performance in the NOR task. Rats received 125 -min exposures to two identical objects and then received either bilateral lesions of the hippocampus or sham surgery $1 \mathrm{~d}, 4 \mathrm{wk}$, or $8 \mathrm{wk}$ after the final exposure. On a retention test $2 \mathrm{wk}$ after surgery, the 1-d and 4-wk hippocampal lesion groups exhibited impaired object recognition memory. In contrast, the 8-wk hippocampal lesion group performed similarly to controls, and both groups exhibited a preference for the novel object. These same rats were then given four postoperative tests using unique object pairs and a 3-h delay between the exposure phase and the test phase. Hippocampal lesions produced moderate and reliable memory impairment. The results suggest that the hippocampus is important for object recognition memory.
\end{abstract}

Recognition memory refers to the ability to judge a previously encountered item as familiar and depends on the integrity of the medial temporal lobe (Squire et al. 2007). Tasks that assess recognition memory (and object recognition memory in particular) have become increasingly useful tools for basic and preclinical research investigating the neural basis of memory (Winters et al. 2008). Perhaps the best known of these tasks is the novel object recognition task (NOR) (also known as the visual pairedcomparison task in studies with humans and monkeys).

Studies of the NOR task in humans with hippocampal damage (McKee and Squire 1993; Pascalis et al. 2004) and in monkeys with selective damage to the hippocampus (Pascalis and Bachevalier 1999; Zola et al. 2000; Nemanic et al. 2004) have resulted in clear and consistent findings. Damage limited to the hippocampus is sufficient to produce impaired recognition memory (Squire et al. 2007, Box 1). In rats and mice, the NOR task has become particularly popular and is currently a benchmark task for assessing recognition memory. Yet despite its widespread use in rodents, the findings are rather mixed. For example, in the rat, although there is agreement that the perirhinal cortex is critically important for normal NOR performance, there is less agreement about the hippocampus (for review, see Winters et al. 2008). Although some of the discrepancies between studies may be attributed to differences in lesion size and in the length of the retention delay (Broadbent et al. 2004), these factors cannot account for all the findings (Squire et al. 2007).

Whereas most studies have investigated the effects of hippocampal lesions on postoperative NOR performance, there is also interest in the effects of hippocampal lesions on memory for previously encountered objects. For a number of tasks, hippocampal lesions produce temporally graded retrograde amnesia, such that memory acquired recently is impaired and memory acquired more remotely is spared (for review, see Squire et al. 2004; Frankland and Bontempi 2005). In the case of the single study of

\section{${ }^{6}$ Corresponding author.}

E-mail reclark@ucsd.edu; fax (858) 534-1569.

Article is online at http://www.learnmem.org/cgi/doi/10.1101//m.1650110. retrograde memory that has involved the NOR task, recognition memory was impaired when a 5-wk interval intervened between training and hippocampal surgery (Gaskin et al. 2003). It remains possible that memory might be spared if a longer delay was imposed between training and surgery.

The aim of the present study was to assess both the anterograde and retrograde effects of hippocampal lesions on recognition memory using the NOR task. To thoroughly assess the effects of hippocampal lesions we used (1) large groups of animals, (2) multiple tests of NOR memory, (3) a scoring method that allowed object preference to be determined on a second-by-second basis during the recognition tests, and (4) a novel training protocol that permitted the evaluation of recognition memory even after a retention interval as long as $10 \mathrm{wk}$.

\section{Results}

\section{Neurohistological findings}

Figure 1 illustrates the smallest (black) and largest (stippled) extents of the hippocampal lesion for each of the training-surgery lesion groups. All rats sustained bilateral damage to all the cell fields of the hippocampus. In cases where the lesion was not complete at a particular level of the hippocampus, the sparing was typically restricted to the most medial aspect of the dorsal dentate gyrus or dorsal CA1 cell field, or the ventral-most region of the hippocampus. In all rats there was damage to the cortex and to the fimbria overlying the dorsal hippocampus, which was associated with the placement of the Hamilton syringe during surgery. There was no evidence of damage to the amygdala. Because impaired performance on the NOR task requires large hippocampal lesions (Broadbent et al. 2004), two rats with less than 75\% total hippocampal damage were removed from further analysis. A summary of the lesion size for each of the hippocampal groups is shown in Table 1.

\section{One-day hippocampal lesion group}

Fifteen rats had lesions to the hippocampus that involved $75 \%-$ $100 \%$ of total hippocampal volume (mean lesion size $=91 \%)$. All 


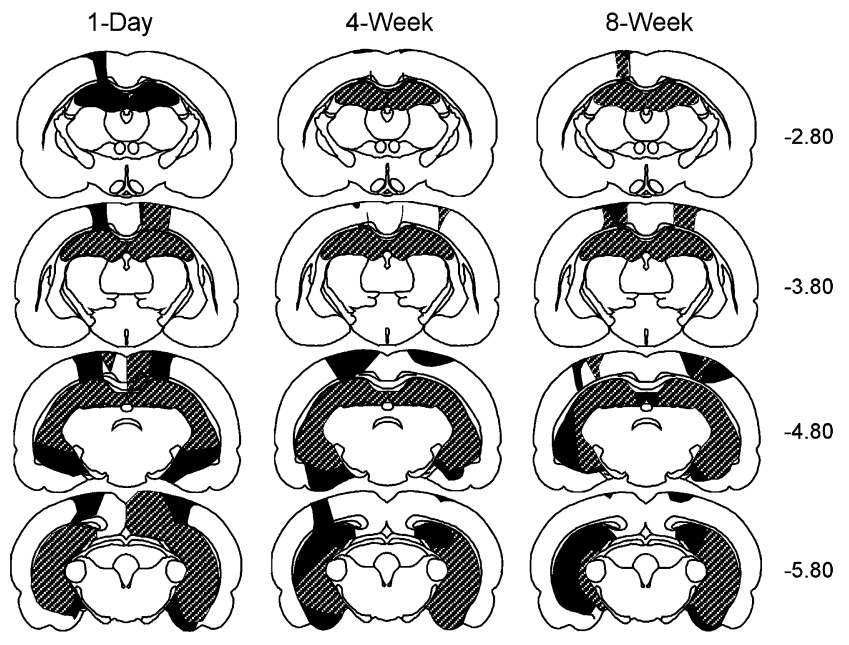

Figure 1. Reconstructions of coronal sections through the hippocampus showing the smallest (black) and largest (stippled) lesions for each of the three hippocampal lesion groups (1-d, 4-wk, and 8-wk trainingsurgery intervals). Numbers (right) represent the distance $(\mathrm{mm})$ posterior to bregma.

rats also had damage to the subiculum $(25 \%-89 \%$, average $=70 \%)$. For eight rats, damage extended beyond the hippocampus to encroach bilaterally upon the entorhinal cortex in the anteriormost sections. In two rats, thinning of the anterior-most region of the perirhinal cortex was noted, but direct damage to the area could not be confirmed.

\section{Four-week hippocampal lesion group}

Fifteen rats had lesions to the hippocampus that involved $87 \%-$ $99 \%$ of total hippocampal volume (mean lesion size $=96 \%$ ). All rats also had damage to the subiculum $(6 \%-88 \%$, average $=61 \%)$. In 14 rats the lesion encroached bilaterally upon the anterior-most regions of the entorhinal cortex, and in one rat there was unilateral entorhinal damage. In one rat, thinning of the anteriormost region of the perirhinal cortex was noted, but direct damage to the area could not be confirmed. the training-surgery intervals ( $1 \mathrm{~d}, 4 \mathrm{wk}$, and $8 \mathrm{wk})$. We calculated the preference for the novel object during the first $15 \mathrm{sec}$ (Fig. 2A) and also during the first $30 \mathrm{sec}$ (Fig. 2B) of cumulative object exploration.

The control groups exhibited a strong preference for the novel object by both measures (one-sample, two-tailed $t$-tests, 15 sec: 1 -d group, $72.6 \pm 4.2 \%$; 4 -wk group, $77.6 \pm 3.9 \%$; 8-wk group, $65.3 \pm 4.3 \%$; 30 sec: 1 -d group, $72.2 \pm 3.1 \%$; 4 -wk group, $75.1 \pm 3.7 \%$; 8-wk group, $62.6 \pm 4.3 \% ; P<0.01$ ). A one-way ANOVA for the training-surgery interval failed to reach significance for the control groups (30 sec: $\left.F_{(2,44)}=3.1, P=0.06\right)$. There was a difference between the 4 -wk and 8-wk control groups $\left(t_{(29)}=\right.$ 2.2, $P=0.04$ ); however, the 1-d group did not differ from either the 4 -wk or 8 -wk groups. Accordingly, though the 8 -wk group performed numerically the poorest, there was not strong evidence for forgetting in intact animals across the three training-surgery intervals.

Neither the 1-d lesion group nor the 4-wk lesion group exhibited a significant preference for the novel object $(15$ sec: Hippocampal 1-d, $61.0 \pm 7.5 \%[t=1.47, P=0.17]$; hippocampal 4 -wk, $55.9 \pm 4.6 \%[t=1.3, P=0.23]$; 30 sec: hippocampal 1 -d, $58.0 \pm 7.3 \%[t=1.0, P=0.29]$; hippocampal 4-wk, $56.4 \pm 3.9 \%[t=$ $1.6, P=0.12]$; chance $=50 \%$ ). In contrast to the 1 -d and 4 -wk group, only the 8-wk hippocampal group exhibited a significant preference for the novel object (15 sec: $64.5 \pm 4.8 \%, P<0.01 ; 30$ sec: $62.1 \pm 5.8 \%, P=0.057)$. Indeed, the 8 -wk lesion group performed similarly to the 8 -wk control group (15 sec: $t_{(28)}=$ $0.13, P>0.1 ; 30$ sec: $\left.t_{(28)}=0.72, P>0.1\right)$.

To compare the groups at each training-surgery interval we first conducted a two-way ANOVA (lesion group by trainingsurgery interval), followed by planned comparisons (Fisher's PLSD) for the 15- and 30-sec time bins. For the 15-sec measure (Fig. 2A), there was a group effect $\left(F_{(1,84)}=7.7, P<0.01\right)$, but no effect of training-surgery interval or interaction $(P>0.12)$. Planned comparisons for the 1 -d group $(P=0.18)$ and 8 -wk group $(P=0.9)$ did not reach significance. The 4-wk lesion group was impaired relative to the control group $(P=0.001)$. For the 30 -sec measure (Fig. 2B), there was also a group effect $\left(F_{(1,84)}=7.9, P<0.01\right)$, but no effect of training-surgery interval or interaction $(P>0.12)$. Planned comparisons for the 1 -d group $(P=0.078)$ and 8 -wk group

\section{Eight-week hippocampal lesion group}

Fourteen rats had lesions to the hippocampus that involved $75 \%-98 \%$ of total hippocampal volume (mean lesion size $=$ $90 \%)$. Eleven rats also had damage to the subiculum $(12 \%-74 \%$, average $=40 \%)$. In seven rats, the lesion encroached bilaterally upon the anterior-most regions of the entorhinal cortex, and three rats had unilateral entorhinal damage. An additional two rats had thinning of the anterior-most region of the perirhinal cortex, but direct damage to the area could not be confirmed, and a further two rats had very minor damage to the dorsal thalamus (one unilateral and one bilateral).

\section{Behavioral findings \\ Postoperative retention of three training-surgery intervals}

Figure $2 \mathrm{~A}, \mathrm{~B}$ shows the preference for the novel object exhibited by the control and hippocampal lesion groups for each of
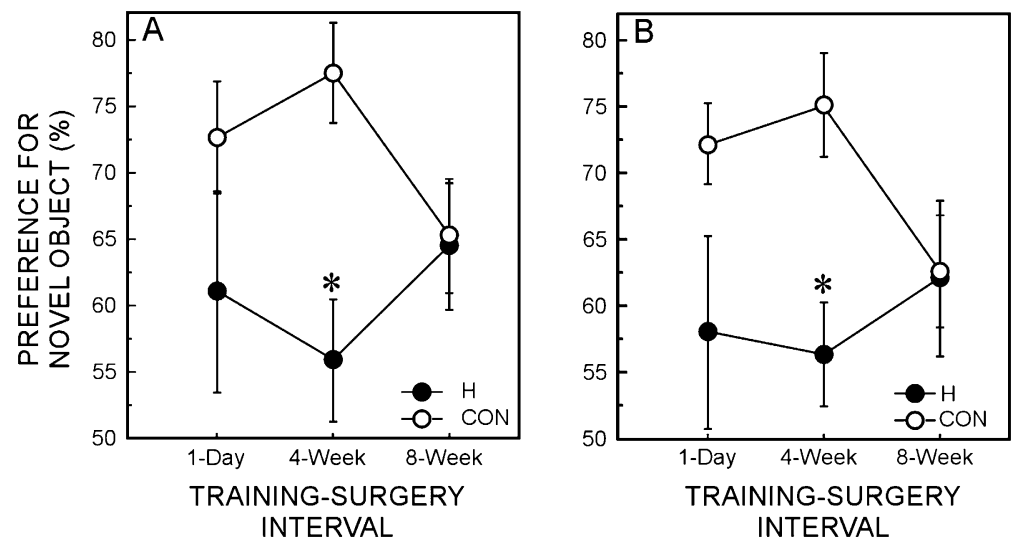

Figure 2. Postoperative retention at three training-surgery intervals. Rats received either sham surgeries or bilateral hippocampal lesions $1 \mathrm{~d}(\mathrm{CON}=16, \mathrm{H}=15), 4 \mathrm{wk}(\mathrm{CON}=16, \mathrm{H}=14)$ or $8 \mathrm{wk}$ ( $\mathrm{CON}=16, \mathrm{H}=14$ ) after training. $(A)$ The cumulative preference for the novel object after $15 \mathrm{sec}$ of object exploration. The control groups at each training-surgery interval exhibited a preference for the novel object, whereas only the 8-wk hippocampal lesion group exhibited this preference. (B) The cumulative preference for the novel object after $30 \mathrm{sec}$ of object exploration. As in $A$, the control groups at each training-surgery interval exhibited a preference for the novel object. However, unlike the $15 \mathrm{sec}$ analysis $(A)$, the 8-wk hippocampal lesion group fell just short of performing above chance $(P=0.057)$. Asterisks indicate group differences $(P<0.05)$. 
$(P=0.95)$ did not reach significance. The 4-wk lesion group was impaired relative to the control group $(P=0.01)$.

We also analyzed performance using both measures, but now excluding those rats that had thinning of the perirhinal cortical region (unilateral or bilateral). Removing these animals did not change the overall pattern of findings. For the 15-sec measure (Fig. 2A), the 1-d hippocampal group and the 4-wk hippocampal group exhibited no preference for the novel object $(P=0.11$ and 0.30 , respectively), and the 4-wk hippocampal group was impaired relative to controls $\left(t_{(27)}=-3.6, P<0.01\right)$. In contrast, the 8 -wk hippocampal group exhibited a significant preference for the novel object $(P<0.05)$ and performed similarly to controls $\left(t_{(26)}=0.3, P=\right.$ 0.8 ). The 30 -sec measure also indicated the 1 -d and 4-wk hippocampal lesion groups performed at chance $(P=0.22$ and 0.19 , respectively). The major difference in results, when the two rats with perirhinal thinning were excluded, was that by the 30-sec measure the 8-wk hippocampal group was not significantly above chance $\left(58.9 \pm 6.3 \% ; t_{(11)}=1.4, P=0.19\right)$.

Last, we conducted a two-way ANOVA involving the hippocampal lesion and control groups and the three training surgery intervals (1-d, 4-wk, and 8-wk). The pattern of findings did not differ when we based the analysis on the 15- or 30-sec time bins, or when we either included or excluded the animals with perirhinal thinning. Accordingly, we present only an analysis from the 30 -sec time bin and include animals with perirhinal thinning. There was an effect for group $\left(F_{(1,84)}=7.9, P<0.01\right)$, but no effect of training-surgery interval $\left(F_{(2,84)}=0.3, P>0.1\right)$ or interaction $\left(F_{(2,84)}=1.9, P>0.1\right)$. A one-way ANOVA for training-surgery interval did not reach significance for the control group $\left(F_{(2,44)}=\right.$ $3.1, P=0.06)$, or the lesion group $\left(F_{(2,40)}=0.3, P=0.78\right)$. Accordingly, we were unable to reliably document forgetting in the control groups or an increase in performance in the lesion group across the three training-surgery intervals.

\section{Object exploration during the familiarization phase}

During the familiarization phase, each rat was allowed to explore the objects for $5 \mathrm{~min}$ on 12 different occasions (three times each day for $4 \mathrm{~d}$ ). We calculated the total amount of time each rat spent exploring the objects during these 5-min periods. The sham groups and the to-be-lesion groups spent a similar amount of total time exploring the objects $(162 \pm 9$ and $169 \pm 8 \mathrm{sec}$, respectively; $\left.t_{(88)}=0.6 ; P>0.1\right)$. Further analysis of exploratory behavior during the familiarization phase revealed two interesting findings. First, although the various groups exhibited a similar amount of total exploration, there was a considerable range of exploration across animals (overall mean $=166 \pm 6 \mathrm{sec}$; range $=$ 64-315 sec). Second, there was a striking reduction in object exploration across the $4 \mathrm{~d}$ of familiarization (day $1=66 \pm 3 \mathrm{sec}$; day $4=29 \pm 2 \mathrm{sec}$ ). This variability allowed us to explore the relationship between amount of exploration during the familiarization phase and subsequent object recognition memory. Would animals that had explored the objects the most thoroughly also exhibit the strongest preference for the novel object (because they had the most experience with the original objects)? Alternatively, perhaps the animals who had explored the objects the least would exhibit the strongest preference for the novel object (because they had learned about the original objects so well that they quickly found them familiar and as a result explored them less overall).

The relationship between object exploration during the familiarization phase and subsequent recognition memory $1 d, 4 w k$, and 8 wk later

Overall (three training-surgery intervals combined for the sham animals), the correlation between the average amount of object exploration during familiarization and novel object preference was $-0.57(P<0.001)$. When the individual training-surgery interval groups were considered separately, negative correlations were observed in all three sham groups (sham 1-d, $r=-0.70, P<$ 0.01 ; 4 -wk, $r=-0.40, P=0.16$; 8 -wk, $r=-0.62, P<0.01)$. Thus, the animals that initially explored the objects the least were the animals that subsequently exhibited the strongest preference for the novel object. As might be expected, for the 1-d and 4-wk hippocampal groups, there was no relationship between the average amount of object exploration during familiarization and subsequent novel object preference (presumably because object preference was at chance for these two groups). However, the 8-wk hippocampal group did exhibit a preference for the novel object, and this group did exhibit a relationship between the average amount of object exploration during familiarization and novel object preference $(r=-0.59, P<0.02)$.

Together, these findings indicate that the less time animals spent exploring the objects during the familiarization phase, the stronger was the novel object preference during the test phase. The implication is that animals that learned about the familiar objects more effectively became less interested in the objects across the multiple familiarization episodes ( 12 episodes across $4 \mathrm{~d}$ ) than animals that learned about the objects less efficiently. The finding that this relationship was found in animals that received hippocampal lesions $8 \mathrm{wk}$ after training (but not in animals that received hippocampal lesions earlier) provides further evidence of normal memory retention in this group.

\section{Postoperative training and testing}

Figure 3A,B shows the percent preference for the novel object for each of the four tests of object recognition and the mean performance for both $15 \mathrm{sec}$ of cumulative object exploration (A) and 30 sec of cumulative object exploration (B). On each day the interval between familiarization and testing was $3 \mathrm{~h}$.

Both the control group and the hippocampal group exhibited a preference for the novel object on each of the four tests of the NOR task. This finding was obtained using both the 15- and 30-sec measures and irrespective of whether rats with apparent perirhinal thinning were included in the analysis $(P<0.05)$. The important finding was that the hippocampal group exhibited less preference for the novel object than the controls across the $4 \mathrm{~d}$ of testing (15 sec: $t_{(89)}=2.4, P<0.05 ; 30$ sec: $\left.t_{(89)}=2.3, P<0.05\right)$. Figure 4 shows the cumulative percent preference for the novel object across $30 \mathrm{sec}$ of object exploration for each group and averaged across the $4 \mathrm{~d}$. A group difference emerged by the fourth second of object exploration $(P<0.05)$, and this difference was maintained throughout the preference test. All data points for both groups were above chance $(P<0.05)$. This analysis indicates that a robust group difference in novel object preference emerged rapidly. The overall pattern of exploration across the 30 -sec test was similar in the two groups, but the control group exhibited a much stronger preference for the novel object than did the hippocampal group.

Importantly, significant group differences were not observed on all of the individual test days. In fact for the 15-sec time bin comparisons, all of the individual test day comparisons failed to reach significance (all $P>0.1$ ). For the 30 -sec time bin analysis, the day 3 and day 1 comparisons were significant or marginally significant, respectively (day $3, t_{(89)}=2.3, P=0.02$; day $1, t_{(89)}=$ $1.9, P=0.059)$, whereas the day 2 and day 4 comparisons were not significant $(P>0.1)$.

\section{Object exploration during the familiarization phase}

The animals with hippocampal lesions spent significantly more time exploring the objects during the 15-min familiarization phases during all four test days (all $P<0.02$ ). The lesion group, on average, spent $80.0 \pm 27.0 \mathrm{sec}$ in each familiarization phase, whereas the control group on average spent $56.0 \pm 20.1 \mathrm{sec}$ in each familiarization phase $\left(t_{(89)}=4.7, P<0.0001\right)$. One interpretation 

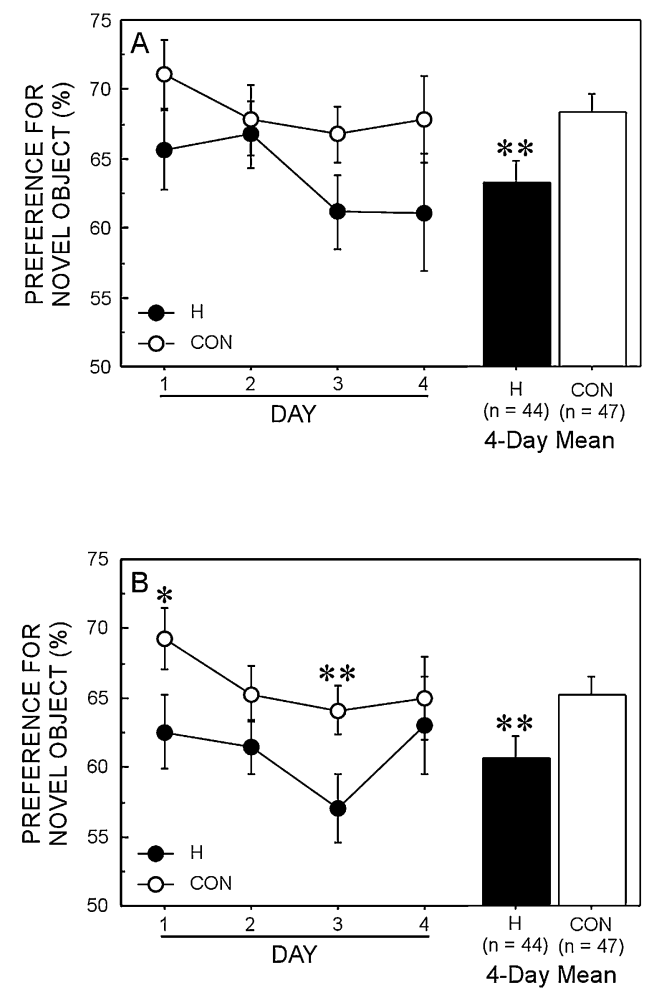

Figure 3. Postoperative training and testing of NOR on four different days in sham operated animals (CON, $n=47)$ and animals with hippocampal lesions $(H, n=44)$. Performance was scored over $15 \mathrm{sec}$ of cumulative object exploration $(A)$ and over $30 \mathrm{sec}$ of cumulative object exploration (B). The left side of each panel (line graphs) shows the percent preference for the novel object on four different days for both the hippocampal lesion group (black circles) and the sham group (white circles). The right side of each panel (bar graphs) shows the 4-d mean for the hippocampal lesion group (black bar) and the sham group (white bar). Asterisks indicate group differences $\left({ }^{*} P=0.059,{ }^{* *} P<0.05\right)$.

of this finding is that rats with hippocampal lesions returned to and explored the objects more than the control group because they had memory impairment. Accordingly, the objects became familiar to rats with hippocampal lesions at a slower rate than control rats (i.e., the objects remained interesting for the lesioned rats for a longer time than they did for the control rats). The increased exploration of the lesion group could also be related to a general increase in activity and exploration, though increased exploration itself could of course be indicative of memory impairment.

\section{The relationship of object exploration during the familiarization phase to recognition memory 3 h later}

We next examined the relationship between the amount of object exploration during the familiarization phase and preference for the novel object during the test phase three hours later. For the control group, there was no overall relationship between familiarization and test phases $(r=0.08, P>0.1)$, although a negative relationship did appear on test day $4(r=-0.38, P<0.01)$. The hippocampal lesion group did exhibit a relationship between object exploration time and novel object preference $(r=-0.33$, $P<0.05)$, but this finding was driven primarily by five poorperforming rats (of the 44 total) that explored the objects more during the familiarization than all the other rats. When these rats were removed from the analysis, the correlation did not approach significance $(P>0.1)$. Thus the relationship between familiariza- tion phase exploration and novel object preference was not as striking when the retention interval was only $3 \mathrm{~h}$ as when the retention interval ranged from $1 \mathrm{~d}$ to $8 \mathrm{wk}$. Though it is unclear what factor might account for this difference, we note that during the familiarization periods for the long-term retention tests, animals accrued much more contact time with the objects than during the single 15-min familiarization phase used for the 3-h delay $\left(166 \pm 6 \mathrm{sec}\right.$ vs. $68 \pm 3 \mathrm{sec}$, respectively, $\left.t_{(88)}=19.2, P<0.01\right)$. Perhaps more experience with the objects is required before a clear relationship between object exploration and subsequent object preference emerges.

\section{Discussion}

Rats were given 5-min exposures to objects on 12 different occasions during $4 \mathrm{~d}$ and then they received either bilateral lesions of the hippocampus or sham surgery $1 \mathrm{~d}, 4 \mathrm{wk}$, or $8 \mathrm{wk}$ after the final 5-min exposure. On a retention test 2 wk after surgery, the 1-d and 4-wk hippocampal lesion groups exhibited impaired object recognition memory. In contrast, the 8-wk hippocampal lesion group performed similarly to controls, and both groups exhibited a significant preference for the novel object. When these same groups of rats were then given four postoperative tests with the same task using unique object pairs, the hippocampal lesion group was moderately impaired when performance was averaged across the four tests.

\section{Postoperative recognition memory}

The present study assessed the anterograde effects of hippocampal lesions on recognition memory using a large group of animals and multiple behavioral tests. As a consequence, we were able to obtain a reliable and robust measure of performance. Even though a significant deficit did not emerge on most individual test days, a reliable impairment was detected when performance was averaged across the four days of testing. The finding that individual NOR tests did not usually detect impaired performance in the animals with hippocampal lesions is important because it suggests that a single test of object recognition memory will often be insufficiently sensitive to reveal impaired recognition memory after hippocampal damage. One notable difference between the present study and others that reported no impairment (see

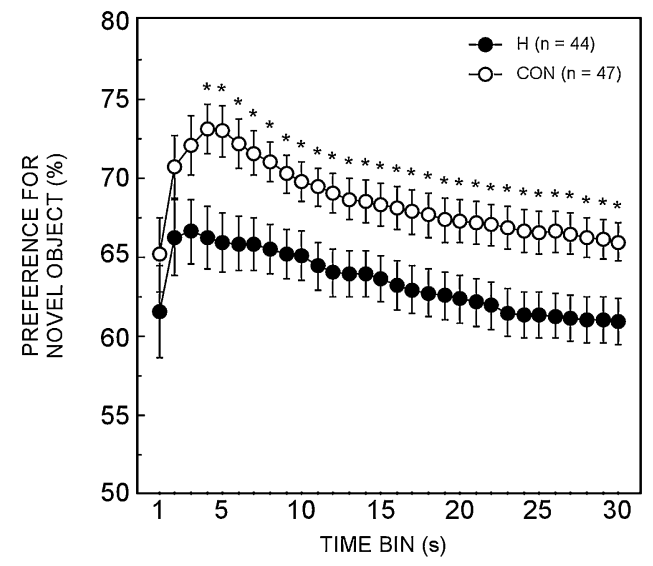

Figure 4. Cumulative percent preference for the novel objects across $30 \mathrm{sec}$ of object exploration averaged across four different days for the hippocampal lesion group and the control group. The pattern of performance indicates that a group difference emerged by the fourth second of object exploration and this difference was maintained throughout the remainder of the preference test. All points for both groups were above chance. Asterisks indicate group differences $(P<0.05)$. 
Table 1. Mean percent of total hippocampal damage (and standard error) for the hippocampal lesion groups (1-d, 4-wk, 8-wk)

Training-surgery interval Hippocampal damage

$1-d$

$4-w k$

$8-w k$

$91.5 \pm 2.3 \%$ (range, $75 \%-100 \%$ )

$96 \pm 1.0 \%$ (range, $87 \%-99 \%)$

$90 \pm 1.7 \%$ (range, $75 \%-98 \%$ )

Winters et al. 2008) is that our study involved a considerably larger number of rats with hippocampal lesions than did any of the earlier studies ( $n=6-17$ rats with hippocampal lesions vs. $n=44$ rats with hippocampal lesions in the present study). Given that the recognition memory impairment in the present study was moderate and that the performance measure in this task can range from $0 \%$ to $100 \%$ even in control animals, detecting impairment may require both multiple test trials and large group sizes. Additionally, we have previously suggested that lesion size and delay length are critical factors influencing whether impaired object recognition memory is detected after hippocampal damage (Broadbent et al. 2004). Hippocampal lesions are more likely to result in impaired recognition memory when the lesion size is large (>75\%; Broadbent et al. 2004) and when the delay length is sufficiently long (>10 min; Clark et al. 2000). Although the present study cannot rule out the possible contribution of a nonmemory performance deficit, previous work using the NOR task has ruled against this possibility by showing a delay-dependent memory impairment following hippocampal lesions (e.g., Clark et al. 2000).

It has been suggested that hippocampal lesions impair object recognition when spatial or contextual cues are visible to rats during testing in large open-field arenas (Winters et al. 2004; Forwood et al. 2005). Further, large arenas might exacerbate impaired exploratory behavior arising from hippocampal lesions, thus resulting in poor performance. Yet, we have found memory impairment after hippocampal lesions both with small arenas that block most external contextual cues and minimize nonobject exploratory behavior (Broadbent et al. 2004), as well as with large arenas where external contextual cues were visible (Clark et al. 2000). Thus, impaired performance on the NOR task need not depend on the presence of contextual cues or impaired exploratory behavior.

To summarize, it appears that impaired recognition memory is most likely detected after hippocampal damage when large numbers of rats are used to assess performance, when recognition memory is assessed with multiple independent tests, when the hippocampal lesion is essentially complete, and when the delays are longer than several minutes.

Although we did observe impairment, it is notable that animals with hippocampal lesions performed above chance on each test day, and that only a moderately severe impairment was observed. Accordingly, structures in addition to the hippocampus must be capable of supporting recognition memory, even across retention intervals as long as $3 \mathrm{~h}$. Thus, although the hippocampus makes an important contribution, structures such as the perirhinal cortex can support performance to some extent (Winters et al. 2004, 2008; Winters and Bussey 2005).

\section{Postoperative retention of preoperative memory}

Rats that received hippocampal lesions $1 \mathrm{~d}$ or $4 \mathrm{wk}$ after training exhibited no memory for the previously encountered familiar object (i.e., performance was at chance). When the lesion was made $8 \mathrm{wk}$ after training, performance was intact. The deficit in the 1-d and 4-wk groups was more severe than the deficit in recognition memory detected postoperatively. This difference in severity might be related to the length of the retention delay for the two conditions ( $3 \mathrm{~h}$ vs. $1 \mathrm{~d}$ or $4 \mathrm{wk}$ ). This observation is also consistent with earlier work showing that, whereas rats trained and tested after hippocampal lesions can exhibit mild memory impairment, or even no memory impairment, rats trained before a hippocampal lesion can exhibit severe impairment (e.g., context fear conditioning, Maren et al. [1997]; visual discrimination learning, Driscoll et al. [2005]; Broadbent et al. [2007]; Epp et al. [2008]; NOR, Gaskin et al. [2003]). This pattern of findings suggests that under normal conditions the hippocampus is critically involved in learning, such that subsequent hippocampal damage severely impairs memory. In contrast, when the hippocampus is damaged before learning, the task can sometimes be acquired using alternative strategies that depend on other brain regions.

The severe retention impairment in animals that received lesions $1 \mathrm{~d}$ or $4 \mathrm{wk}$ after training is consistent with the single previous study that evaluated postoperative retention of preoperative memory using the NOR task (Gaskin et al. 2003). In that study, rats received hippocampal lesions either $48 \mathrm{~h}$ or $5 \mathrm{wk}$ after training, and recognition memory was severely impaired.

In contrast to the findings in the 1-d and 4-wk groups, remote memory for the familiar object was intact when hippocampal lesions were made $8 \mathrm{wk}$ after training. However, this finding should be interpreted with caution. The strongest evidence for temporal gradients of retrograde amnesia comes from studies where the performances of the lesion groups differ as a function of training-surgery interval, such that remote-memory performance is significantly better than recent-memory performance (e.g., Clark et al. 2002). In the present case, performance in the 8-wk group was significantly above chance (unlike performance in the 1-d and 4-wk groups), but the 8-wk group did not perform significantly better than the 1-d or 4-wk lesion groups. Further, the interaction term from a two-way ANOVA for lesion group and training-surgery interval was not significant. Accordingly, we cannot reach an unambiguous, strong conclusion regarding temporal gradients from these findings. Although this feature of the data tempers our conclusions about the sparing of remote memory after hippocampal lesions, there is an additional reason to suppose that the 8-wk group is different from the 1-d and 4-wk groups. Specifically, the 8-wk group, like the control group, exhibited a negative relationship between total exploration time during the familiarization phase and NOR performance (8-wk hippocampal group, $r=-0.59$; control group, $r=-0.62$ ). That is, the animals that explored the objects most during the familiarization phase were the ones who later exhibited the weakest preference for the novel object. The 1-d and 4-wk lesion groups did not exhibit this relationship.

It is worth mentioning another report that found a positive relationship, rather than a negative relationship, between exploration time during the familiarization phase and NOR performance (Albasser et al. 2009). The difference between that study and the present study is that in Albasser et al. (2009), rats had restricted exposure times during familiarization training $($ mean $=$ $64 \mathrm{sec}$ ), whereas in our study rats had extended exposure times $($ mean $=162 \mathrm{sec})$. Interestingly, in both studies, the animals that accumulated 80-120 sec of object exploration during the familiarization phase performed the best in the subsequent NOR test. Animals that explored less than this amount performed poorly on the NOR test (Albasser et al. 2009), and animals that explored more than this amount also performed poorly (present study). We suggest that poor NOR performance can occur either because animals insufficiently explore the objects during the familiarization phase (Albasser et al. 2009) or because some animals tend to encode the objects inefficiently. These rats will tend to explore the objects more during the familiarization phase and perform poorly during the test. 
In summary, the present findings indicate that the hippocampus is important for object recognition memory as assessed by the NOR task. Hippocampal lesions produce a moderate and reliable anterograde memory impairment. This finding appeared to depend on using large groups of animals and multiple tests, which may explain why several studies have not observed impaired recognition memory in the NOR task after hippocampal damage (e.g., Winters et al. 2004; Forwood et al. 2005; Mumby et al. 2005). Furthermore, the findings suggest that the hippocampus may play a time-limited role in memory for this task, though our data on this point do not permit a strong conclusion. Together with previous work involving rodents, nonhuman primates, and memory-impaired patients, the present study provides strong support for the conclusion that normal recognition performance depends on the integrity of the hippocampus (Squire et al. 2007).

\section{Methods}

\section{Subjects}

Subjects were 94 male, Long-Evans rats weighing between 300 and $350 \mathrm{~g}$ at the beginning of the study. Rats were individually housed and maintained on a 12:12 h light:dark cycle. Food and water were freely available. Rats were randomly assigned to receive either bilateral lesions of the hippocampus $(\mathrm{H})$ or sham surgeries $(\mathrm{CON})$. Surgery occurred either $1 \mathrm{~d}$ after completion of training $(\mathrm{H}=16 ; \mathrm{CON}=16) ; 4 \mathrm{wk}$ after training $(\mathrm{H}=15 ; \mathrm{CON}=16)$ or $8 \mathrm{wk}$ after training $(\mathrm{H}=15 ; \mathrm{CON}=16)$.

\section{Apparatus}

The NOR task was conducted in an opaque plastic box measuring $35 \mathrm{~cm} \times 41.5 \mathrm{~cm} \times 50 \mathrm{~cm}$ high. Stimuli consisted of ceramic or plastic objects that varied in color and size (width $=7.6-8.9 \mathrm{~cm}$; height $=7.5-12.7 \mathrm{~cm}$ ). Three identical copies of each object were available. The objects were secured to the floor of the box using Velcro strips situated $\sim 9 \mathrm{~cm}$ apart. A video camera mounted on the wall directly above the box was used to record the testing session for off-line analysis. Overhead fluorescent lighting illuminated the box.

\section{Procedure}

\section{Habituation}

Rats were acclimated to the testing room and chamber for two consecutive days prior to testing ( $45 \mathrm{~min}$ in the testing room and 5 min to explore the empty box).

\section{Object familiarization}

Rats were given $4 \mathrm{~d}$ of familiarization with the two identical sample objects (three exposures each day). On each day of testing, rats were acclimated to the testing room for $45 \mathrm{~min}$ and then placed in the empty box for $1 \mathrm{~min}$. Then the rat was removed and two identical objects were placed centrally $9 \mathrm{~cm}$ apart. The rat was then placed back in the box and allowed to explore for $5 \mathrm{~min}$. Once all rats in the group were familiarized with the objects, the same procedure was repeated two more times on the same day (mean interval between daily exposures about $60 \mathrm{~min}$ ). Altogether, rats were repeatedly exposed to the same two identical objects for a total of 12,5-min familiarization exposures distributed across $4 \mathrm{~d}$. The same sample objects were used for each of the three training-surgery interval groups.

\section{Surgery}

Surgery designed to remove the entire hippocampus was conducted 24-36 h (designated as the 1-d group), $4 \mathrm{wk}$, or $8 \mathrm{wk}$ after the final familiarization day. Anesthesia was maintained throughout surgery with isoflurane gas $(0.8 \%-2.0 \%$ isoflurane delivered in $\mathrm{O}_{2}$ at $1 \mathrm{~L} / \mathrm{min}$ ). The rat was placed in a Kopf stereotaxic instrument, and the incisor bar was adjusted until Bregma was level with Lambda. For the lesion group $(\mathrm{H})$, bilateral excitotoxic hippocampal lesions were produced by local microinjections of ibotenate acid (IBO; Biosearch Technologies). IBO was dissolved in $0.1 \mathrm{M}$ phosphate-buffered saline to provide a solution with a concentration of $10 \mathrm{mg} / \mathrm{mL}, \mathrm{pH} 7.4$. IBO was injected at a rate of $0.1 \mu \mathrm{L} / \mathrm{min}$ with a $10 \mu \mathrm{L}$ Hamilton syringe mounted on a stereotaxic frame and held with a Kopf Microinjector (model 5000). The syringe needle was lowered to the target coordinate and left in place for $1 \mathrm{~min}$ before beginning the injection. Following the injection, the syringe needle was left in place for a further 2 min to reduce the spread of IBO up the needle tract. For the lesion group, a total of $0.51 \mu \mathrm{L}$ of IBO was injected into 18 sites within each hippocampus (for coordinates, see Clark et al. 2000). The procedure for the sham-operated control $(\mathrm{CON})$ group was the same as for the lesion groups, with the exception that the dura was not punctured, the syringe needle was not lowered into the cortex, and no IBO was injected. Once awake and responsive, each rat was returned to its home cage in the colony room for a 14-d recovery period.

\section{NOR test}

Retention testing began 15-16 d following surgery. Each rat was first rehabituated to the testing area by being placed in the empty box for $1 \mathrm{~min}$. The rat was then removed, two objects (one novel object and a copy of the object from the familiarization phase) were placed in the box and the rat was allowed to explore the objects for $10 \mathrm{~min}$. Object exploration was later scored from video recordings of each trial by an experimenter who was blind to the group membership of the rats during testing and during off-line data analysis. Object exploration was scored when the rat's nose was within $1 \mathrm{~cm}$ of the object and the vibrissae were moving (see Clark et al. 2000). Object exploration was not scored when the rat used the object to rear upward with the nose of the rat facing the ceiling. Preference for the novel object was expressed as the percent time that a rat spent exploring the novel object (compared to the familiar object). Which object served as the novel object and the left/right position of the novel object were counterbalanced within each group.

To characterize postoperative retention, preference for the novel object was evaluated during the first $15 \mathrm{sec}$ of cumulative object exploration, as well as during the first $30 \mathrm{sec}$ of cumulative object exploration. Previous work has shown (e.g., Clark et al. 2000) that the strongest novel object preference scores tend to occur early in the test phase (i.e., during the time when the novel object is still relatively novel-as opposed to later in the test trial when continuing exploration of the novel object makes it more familiar).

\section{Anterograde memory testing}

Beginning 5-16 d after the retention test, the same rats were given four additional NOR tests. Rats received one session per day for $4 \mathrm{~d}$ with novel pairs of objects in each session. Each daily session was conducted as follows: The rat was first acclimated to the testing room for $45 \mathrm{~min}$ and then placed in the empty box for $1 \mathrm{~min}$. Then the rat was removed, and two identical objects were placed centrally $9 \mathrm{~cm}$ apart. The rat was then allowed to explore the box and the objects for $15 \mathrm{~min}$. After a delay of $3 \mathrm{~h}$, the rat was returned to an empty box for $1 \mathrm{~min}$, and then reintroduced to the box in the presence of two objects: a novel object and a copy of the previously encountered object. Preference for the novel object was expressed as the percent time that a rat spent exploring the novel object (compared to the familiar object).

\section{Histology}

At completion of testing, the rats were administered an overdose of sodium pentobarbital and perfused transcardially with buffered $0.9 \% \mathrm{NaCl}$ solution followed by $10 \%$ formaldehyde solution (in $0.1 \mathrm{M}$ phosphate buffer). The brains were then removed and cryoprotected in $20 \%$ glycerol $/ 10 \%$ formaldehyde. Coronal sections $(50 \mu \mathrm{m})$ were cut with a freezing microtome beginning at the level of the anterior commissure and continuing caudally through the length of the hippocampus. Every fifth section was mounted 
and stained with thionin to assess the extent of the lesions. Lesion size estimates were obtained by calculating the percent damage in $1 \mathrm{~mm}$ increments through the anterior-posterior extent of the hippocampus ( 4 sections, from -2.80 to $-5.80 \mathrm{~mm}$ from bregma) (Paxinos and Watson 1998). Each section was assessed under magnification, and the tissue was considered damaged if it was absent or necrotic (i.e., hippocampal tissue was present, but there was no evidence of Nissl staining, or the tissue was gliotic). The region damaged was drawn onto a control template for each section, and the area of damage was calculated using an automated tool in a computer graphics program (Canvas 8, Deneba). The area of damage was then summed across sections and calculated as a percentage of the total control hippocampal area.

\section{Acknowledgments}

This work was supported by the Medical Research Service of the Department of Veterans Affairs, the National Institute of Mental Health, the Metropolitan Life Foundation, National Institute of Aging grant (P50 AG05131), the National Science Foundation (SBE 0542013 and 237053), the James S. McDonnell Foundation, the Kavli Foundation, and a NSF grant (\#SBE-0542013) to the Temporal Dynamics of Learning Center, a NSF Science of Learning Center. We thank Susan Davis, Laura Johnson, and Brittany Masatsugu for their assistance.

\section{References}

Albasser MM, Davies M, Futter JE, Aggleton JP. 2009. Magnitude of object recognition deficit associated with perirhinal cortex damage in rats: Effects of varying the lesion extent and the duration of the sample period. Behav Neurosci 123: 115-124.

Broadbent NJ, Squire LR, Clark RE. 2004. Spatial memory, recognition memory, and the hippocampus. Proc Natl Acad Sci 101: 14515-14520.

Broadbent NJ, Squire LR, Clark RE. 2007. Rats depend on habit memory for discrimination learning and retention. Learn Mem 14: 145-151.

Clark RE, Zola SM, Squire LR. 2000. Impaired recognition memory in rats after damage to the hippocampus. J Neurosci 20: 8853-8860.

Clark RE, Broadbent NJ, Zola SM, Squire LR. 2002. Anterograde amnesia and temporally-graded retrograde amnesia for a nonspatial memory task following lesions of hippocampus and subiculum. J Neurosci 22: 46634669.

Driscoll I, Howard SR, Prusky GT, Rudy JW, Sutherland RJ. 2005. Seahorse wins all races: Hippocampus participates in both linear and non-linear visual discrimination learning. Behav Brain Res 164: 29-35.

Epp J, Keith JR, Spanswick SC, Stone JC, Prusky GT, Sutherland RJ. 2008. Retrograde amnesia for visual memories after hippocampal damage in rats. Learn Mem 15: 214-221.
Forwood SE, Winters BD, Bussey TJ. 2005. Hippocampal lesions that abolish spatial maze performance spare object recognition at delays of up to 48 hours. Hippocampus 15: 347-355.

Frankland PW, Bontempi B. 2005. The organization of recent and remote memories. Nat Rev Neurosci 6: 119-130.

Gaskin S, Tremblay A, Mumby DG. 2003. Retrograde and anterograde object recognition in rats with hippocampal lesions. Hippocampus 13: 962-969.

Maren S, Aharonov G, Fanselow MS. 1997. Neurotoxic lesions of the dorsal hippocampus and Pavlovian fear conditioning in rats. Behav Brain Res 110: $436-442$.

McKee RD, Squire LR. 1993. On the development of declarative memory. J Exp Psychol Learn Mem Cogn 19: 397-404.

Mumby DG, Tremblay A, Lecluse V, Lehmann H. 2005. Hippocampal damage and anterograde object-recognition in rats after long retention intervals. Hippocampus 15: 1050-1056.

Nemanic S, Alvarado MC, Bachevalier J. 2004. The hippocampal/ parahippocampal regions and recognition memory: Insights from visual paired comparison versus object-delayed nonmatching in monkeys. J Neurosci 24: 2013-2026.

Pascalis OP, Bachevalier J. 1999. Neonatal aspiration lesions of the hippocampal formation impair visual recognition memory when assessed by paired-comparison task but not by delayed nonmatchingto-sample task. Hippocampus 9: 609-616.

Pascalis O, Hunkin NM, Holdstock JS, Isaac CL, Mayes AR. 2004. Visual paired comparison performance is impaired in a patient with selective hippocampal lesions and relatively intact item recognition. Neuropsychologia 42: 1293-1300.

Paxinos G, Watson C. 1998. The rat brain in stereotaxic coordinates, 4 th ed. Academic, San Diego, CA.

Squire LR, Clark RE, Bayley P. 2004. Medial temporal lobe functions and memory. In The cognitive neurosciences, 3rd ed. (ed. M Gazzaniga), pp. 691-708. MIT, Cambridge, MA.

Squire LR, Wixted JT, Clark RE. 2007. Recognition memory and the medial temporal lobe: A new perspective. Nat Rev Neurosci 8: 872-883.

Winters BD, Bussey TJ. 2005. Glutamate receptors in perirhinal cortex mediate encoding, retrieval, and consolidation of object recognition memory. J Neurosci 25: 4243-4251.

Winters BD, Forwood SE, Cowell RA, Saksida LM, Bussey TJ. 2004. Double dissociation between the effects of peri-postrhinal cortex and hippocampal lesions on tests of object recognition and spatial memory: Heterogeneity of function within the temporal lobe. J Neurosci 24: 5901-5908.

Winters BD, Saksida LM, Bussey TJ. 2008. Object recognition memory: Neurobiological mechanisms of encoding, consolidation and retrieval. Neurosci Biobehav Rev 32: 1055-1070.

Zola SM, Squire LR, Teng E, Stefanacci L, Buffalo EA, Clark RE. 2000. Impaired recognition memory in monkeys after damage limited to the hippocampal region. J Neurosci 20: 451-463.

Received September 28, 2009; accepted in revised form October 5, 2009. 


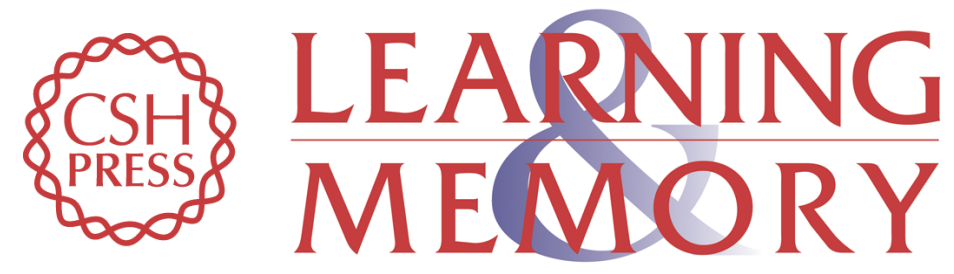

\section{Object recognition memory and the rodent hippocampus}

Nicola J. Broadbent, Stephane Gaskin, Larry R. Squire, et al.

Learn. Mem. 2010, 17:

Access the most recent version at doi:10.1101//m.1650110

References This article cites 21 articles, 9 of which can be accessed free at: http://learnmem.cshlp.org/content/17/1/5.full.html\#ref-list-1

License

Email Alerting Receive free email alerts when new articles cite this article - sign up in the box at the Service top right corner of the article or click here. 